\title{
El fútbol como desafío étnico-racial y nacional: tensiones alrededor de su práctica en Otavalo (Andes ecuatorianos)
}

\author{
Jérémie Voirol ${ }^{1}$
}

Recibido: 16 de diciembre de 2016 / Aceptado: 28 de julio de 2017

\begin{abstract}
Resumen. Este artículo se interesa por el tema del fútbol en el medio indígena en la región de Otavalo, tema todavía poco abordado en antropología y en las ciencias sociales. El objetivo es, por una parte, mostrar la reapropiación local de un juego "global" - a través del intento de reconstrucción histórica de la aparición del fútbol en esta región, de la atención a la conectividad, de su dimensión moral y de la producción de la masculinidad-y, por otra, reubicar esta práctica local en un contexto más amplio. De esta manera, la aprehensión del fútbol por los indígenas otavalos resalta tensiones entre la reproducción de una visión racial de la sociedad ecuatoriana y el desafío de esta sociedad desigual, a través de la reapropiación de características étnico-raciales y de la competición deportiva. Por lo tanto, el fútbol en Otavalo constituye un medio, para los indígenas, de integrarse al mundo contemporáneo y de proponer una concepción alternativa de la nación, más allá de la del "mestizaje" y del multiculturalismo.

Palabras clave: Fútbol; contemporaneidad; moral; masculinidad, indígenas; etnicidad; raza; nación; discriminación; Otavalo; Ecuador.
\end{abstract}

[en] Soccer as ethnic-racial and nation challenge: tensions about the practice in Otavalo (Ecuadorian Andes)

\begin{abstract}
This article is interested in the soccer topic in the indigenous milieu in the region of Otavalo, topic that is still little addressed by anthropologists and social scientists. The aim is, on one hand, to show the local reappropriation of a "global" game - through the attempt of historical reconstruction of soccer appearance in this region, the attention to connectedness, the morality dimension and the production of masculinity - and, on the other, to relocate the local practice of soccer in Otavalo in a broader context. In this way, the grasping of soccer by indigenous people from Otavalo highlights tensions between the reproduction of a racial vision of the Ecuadorian society and the challenge of this unequal society, through reappropriation of ethnic-racial features and through sport competition. Consequently, soccer in Otavalo constitutes a means, for indigenous people, to integrate the contemporary world and to offer an alternative conception of the nation, beyond the "mestizaje" and multiculturalist ones.
\end{abstract}

Keywords: Soccer; contemporaneity; morality; masculinity; indigenous people;ethnicity; race; nation; discrimination; Otavalo; Ecuador.

Sumario: 1. Surgimiento y desarrollo del fútbol en Otavalo 2. Producción de la masculinidad y "moral en acto" en la práctica del fútbol en Otavalo 3. El fútbol como reafirmación indígena: surgimiento de lo racial 4. El fútbol como reproducción y desafío de la desigualdad racial en la sociedad ecuatoriana 5. El fútbol como ingrediente de pertenencia nacional para los otavalos 6 . Conclusión

1 Laboratorio de antropología cultural y social, Universidad de Lausanne, Suiza, Granada Centre for Visual Anthropology, Universidad de Manchester, Reino Unido.jeremie_voirol@yahoo.fr 
Cómo citar: Voirol, J. (2018). El fútbol como desafío étnico-racial y nacional: tensiones alrededor de su práctica en Otavalo (Andes ecuatorianos), en Revista de Antropología Social 27(1), 73-94.

El propósito de este artículo es generar una reflexión y un interés sobre el tema del deporte en el medio indígena ${ }^{2}$. A pesar de la popularidad del fútbol, principalmente en este contexto, se encuentran todavía pocos estudios en ciencias sociales sobre esta temática. Incluso, esta área de vacancia refleja el desprecio que ha tenido durante mucho tiempo la academia acerca del fútbol en general, a pesar de su importancia en muchas partes del mundo (Miller, 2007: 18; Wittersheim, 2014). Los antropólogos Da Matta (1982), de Brasil, y Archetti (1995), de Argentina, constituyen dos de los precursores en estudios sobre este deporte en el contexto sudamericano (Miller, 2007).

En el caso de los indígenas del sub-continente, tenemos que esperar al siglo XXI para encontrar investigaciones sobre deporte, a pesar de la práctica del fútbol por lo menos desde los años 1940 en varias partes (Kowii, 2006; Acuña Delgado, 2010; Bonifacio, 2011; Walker, 2013). Por lo tanto, como lo subraya Sanjek (2014: 86-88), de manera general parece que los antropólogos que investigaban sobre los indígenas sudamericanos se han preocupado más en dedicarse a las prácticas que ellos mismos consideraron como "tradicionales", buscando conectarlas con el periodo prehispánico, perspectiva calificada de "orientalismo" por Ferraro (2004: 43) refiriéndose al trabajo de Edward Said.

Dada esta situación, quisiera intentar mostrar, en primer lugar, cómo emergió la práctica futbolera en la región indígena de Otavalo, en los Andes ecuatorianos, donde he llevado en total más de un año y medio de investigación antropológica de campo sobre fiestas. A pesar de que la historiografía del juego en esta área necesitaría un estudio más profundo, mi bosquejo permitirá establecer algunas bases en dicho ámbito. Mi objetivo es aprehender cómo un deporte se difunde y se desarrolla en un medio específico. El fútbol me llamó la atención porque constituye la actividad principal de varias fiestas, de las cuales las más importantes son el Pawkar Raymi — "Fiesta del Florecimiento"_ _ de la comunidad de Peguche con su "Mundialito indígena" ${ }^{3}$ y el Carnaval de la comunidad vecina de Agato.

El fútbol constituye una práctica contemporánea que se juega en la mayor parte del planeta. Dada la popularidad de los eventos futboleros en la zona indígena de Otavalo, me interesa, en segundo lugar, la reapropiación local de este deporte, es

2 La investigación de campo que forma la base de este artículo se ha beneficiado de un apoyo financiero de la Société Académique Vaudoise — Lausanne, Suiza_, de la sección de antropología de la École des Hautes Études en Sciences Sociales — París - y del Instituto de ciencias sociales de la Universidad de Lausanne — SuizaAprovecho para agradecer por su ayuda financiera en diferentes periodos de mi investigación de campo la Société Académique Vaudoise —agosto del 2010-julio del 2011—, la sección de antropología de la EHESS — junio-julio del 2008 - y el Instituto de ciencias sociales de la Universidad de Lausanne — enero-febrero del 2012 - También doy las gracias a la FLACSO-Ecuador por haberme acogido como investigador visitante de agosto del 2010 a julio del 2011. Agradezco igualmente a Cristian Terry y a Lizardo Herrera por la lectura y los comentarios de este artículo, así como a los dos evaluadores anónimos de esta revista.

3 Según Kowii (2006: 70), la prensa nacional empezó a llamar al torneo con dicha apelación por los nombres de algunos equipos, conformados por migrantes indígenas de Otavalo que los llaman por el lugar de residencia - como Barcelona, Holanda, Boca Juniors (Buenos Aires), MetroStars (Nueva York). Hay que precisar que, desde el año 1998, el equipo ganador recibe una réplica de la Copa del Mundo FIFA (Muenala, 2009). Wibbelsman (2004) y Ordóñez (2008) abordan la fiesta del Pawkar Raymi de Peguche, sin embargo, no desarrollan una reflexión específicamente sobre el fútbol en el medio indígena. 
decir la producción de una "localidad" a través de este. De esta manera, resaltaré el papel de los cuerpos en la producción del género, así como de una moral que surge de la práctica misma del fútbol y que destaca la idea de un cuerpo sano, idea opuesta a la del consumo de alcohol, y promovida particularmente por los miembros - minoritarios - de las iglesias evangélica y mormona. La bebida embriagante, tan importante en la sociabilidad festiva católica, no es adecuada en el contexto deportivo.

En tercer lugar, intentaré reubicar la práctica del fútbol en Otavalo de manera más amplia. En efecto, mostraré cómo el deporte puede establecer conexiones con la idea de "indigeneidad" — como "label" étnico-racial (Becker, 1963)—, de nación y de contemporaneidad ${ }^{4}$. Basándome en mis datos etnográficos del "Mundialito indígena" del Pawkar Raymi de Peguche, argumentaré que el fútbol participa en la construcción de un "reagrupamiento" indígena - "reagrupamiento" en el sentido de Latour (2007) - . Esta construcción reproduce, y al mismo tiempo desafía, una concepción del espacio nacional desigual, pensado en términos de "razas" - "blancos", "indígenas" y "negros"-, concepción que procede de la "ideología del mestizaje" adoptada por la mayoría de los Estados latinoamericanos desde el siglo XIX y que promueve al "mestizo" como garante de la unidad nacional. Esto implica en realidad una homogeneización hacia un "blanqueamiento", es decir, la valorización de la herencia europea y el descrédito de lo que es identificado como indígena o afrodescendiente $^{5}$ (ver Stutzman, 1981; Whitten, 1981; Huarcaya, 2014; Rahier, 2014).

Finalmente, el fútbol aparecerá también como una fuente para imaginar una nación, por parte de los indígenas otavalos, que brinda una alternativa a la de los seguidores del mestizaje y del multiculturalismo — distintas "culturas" como parte de la nación - que fue adoptada en la constitución de 1998. Constituirá de igual manera, para los hombres otavalos particularmente, otro modo de conectarse al mundo contemporáneo y de sentirse parte de él.

Cabe destacar aquí que los otavalos constituyen un pueblo indígena con varias peculiaridades con relación a otras poblaciones autóctonas de las Américas. En efecto, en el transcurso del siglo XX, desarrollaron, con un cierto éxito, aunque desigual, actividades de producción y de comercialización de artículos artesanales — principalmente textiles-, particularmente a través del mercado de la ciudad de Otavalo - que atrae un sinnúmero de turistas nacionales y extranjeros - y de prácticas migratorias, temporales y permanentes, primero en América Latina y después especialmente a Estados Unidos, Europa y Japón (Meisch, 2002). Estas actividades han superado la centralidad de la agricultura del principio del siglo XX (ColloredoMansfeld 1999). La zona rural de la región, ubicada en la provincia de Imbabura a menos de $100 \mathrm{~km}$ al norte de Quito, está poblada mayormente por indígenas kichwahablantes, mientras que la ciudad de Otavalo — más de 40.000 habitantes - lo está

4 En este artículo, utilizo categorías "étnicas" — refiriéndose a una "cultura"— y "raciales" —ligadas a ideas sobre los cuerpos y la filiación_- principalmente émicas. En efecto, me intereso por el uso que hacen de estas los diferentes actores sociales, es decir, cómo les dan significado - el contenido de dichas categorías no es predefinido - y cómo se auto-identifican y exo-asignan a los otros (Amselle y M'Bokolo, 1985). Sin embargo, este juego de significaciones implica tomar en cuenta la materialidad de los cuerpos, la historia, las relaciones políticas (Wade, 2000, 2015; Canessa, 2012) y la particularidad de las situaciones (Moerman, 1994). Los movimientos indígenas y el Estado reconocen a los indígenas de la región de Otavalo como "otavalos". Sin embargo, los otavalos se autodenominan más como "runa" — "indígena" en kichwa, su idioma materno- o como "otavaleño/a".

5 Por esta razón, voy a utilizar también la categoría "blanco-mestizo", como es habitual, de hecho, en la literatura de ciencias sociales que trata del Ecuador (ver, por ejemplo, Whitten, 1981; Rahier, 2014). 
por blanco-mestizos. Sin embargo, un número no despreciable de comerciantes indígenas exitosos han conquistado el centro de esta localidad.

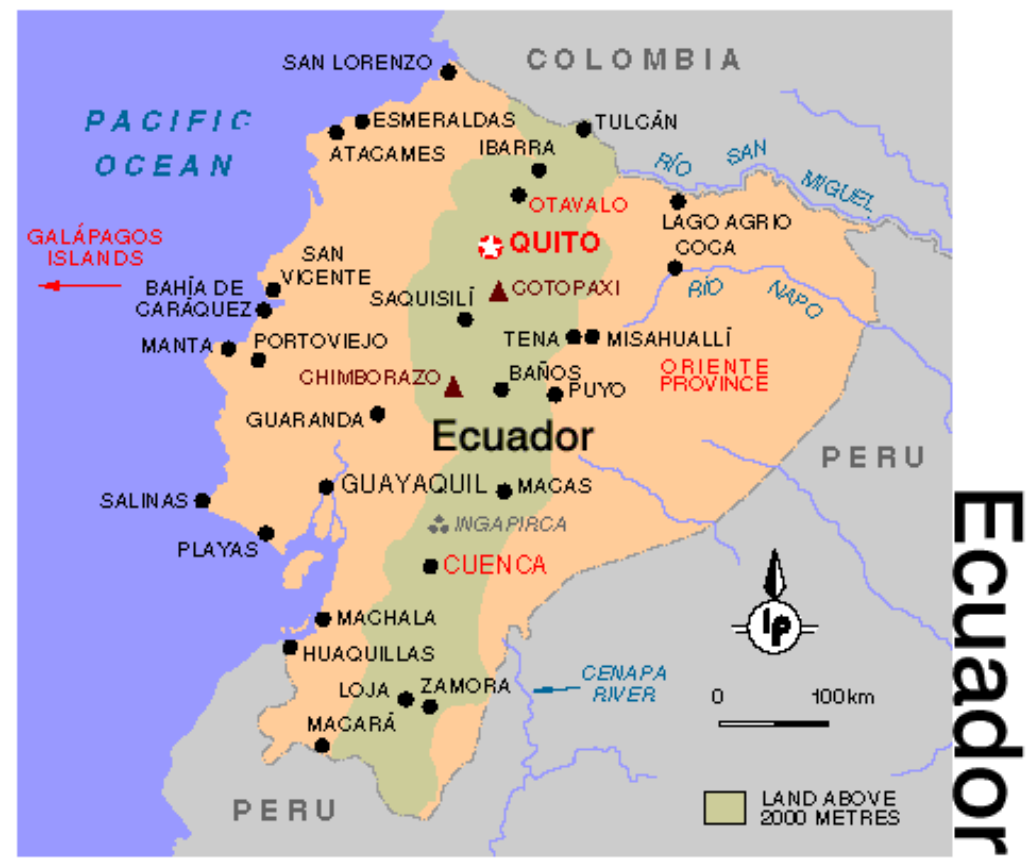

Mapa 1. Ubicación de Otavalo (fuente: http://www.turkey-visit.com).

\section{Surgimiento y desarrollo del fútbol en Otavalo}

Miller (2007: 3) afirma que los marinos y migrantes ingleses trajeron el fútbol a Sudamérica en la segunda mitad del siglo XIX (ver, para Argentina, Archetti, 1995: 76, 80-81; para Perú, Wood, 2007: 127). En efecto, en esa época el comercio y las inversiones británicas se desarrollaron rápidamente en esta parte del mundo — sobre todo en Brasil y en el cono sur-, generando una fuerte migración de jóvenes ingleses que fueron contratados en los bancos, así como en las compañías de ferrocarril y de tranvía, y que fundaron, entre otros, clubes de deporte (Miller, 2007: 4) ${ }^{6}$.

En Ecuador, el fútbol llegó también por los puertos, principalmente por el de Guayaquil en la década de 1890 (Bustamante, 2006: 38; Carrión, 2006: 66); los hermanos Wright, de nacionalidad británica, organizaron los primeros partidos registrados y los primeros clubes fueron fundados al inicio del siglo XX, primero en Guayaquil —en 1900 el Club Deportivo Guayaquil—, después en el resto del país (Bustamante, 2006: 38, 41, 45).

En Argentina, el primer club de fútbol, el Buenos Aires Football Club — división del Buenos Aires Cricket Club- fue fundado en 1867 y numerosos otros clubes aparecieron después de 1880, la mayoría proviniendo de las escuelas inglesas (Archetti, 1995: 80). De esa época son también los clubes de la elite británica que juegan al fútbol en Perú, principalmente en Lima (Wood, 2007: 127-128). 
El fútbol se desarrolló en Sudamérica a partir de esta época, en la misma medida que en Europa meridional, y su control escapó poco a poco de las manos de los ingleses (Miller, 2007: 3-6). Además, el primer torneo internacional de selecciones nacionales tuvo lugar en este sub-continente, el Campeonato Sudamericano de Selecciones - Copa América desde 1975-, en 1916 en Argentina ${ }^{7}$ y el primer Mundial se organizó en Uruguay en 1930, las dos competiciones ganadas por este mismo país (Archetti, 1995: 81; Miller, 2007: 7-8). Por lo tanto, el fútbol se constituyó primero como un deporte de elite — clubes formados por los británicos-, difundiéndose sin embargo rápidamente en los sectores obreros urbanos y otorgándole una gran popularidad en todo el continente sudamericano (Miller, 2007: 6-7).

No es fácil saber cómo y cuándo el medio indígena empezó a practicar el fútbol. Walker (2013) declara que este deporte está relacionado con la construcción de la nación y con el ideal de modernidad en América del Sur. La ambigüedad, en el pasado, de los Estados latinoamericanos con respecto al papel de las poblaciones indígenas en la nación moderna - entre voluntad de asimilación y exclusión (Whitten, 1981, 2003; Huarcaya, 2014) — no permite establecer hipótesis. Además, como he dicho, los antropólogos se interesaron por el tema sólo recientemente. Sin embargo, Bonifacio (2011), basándose en el libro Condiciones para el establecimiento de misiones en Paraguay publicado en 1954 por la eslovena Branislava Susnik, hace remontarse a los años 1940 la emergencia del fútbol en el caso de los indígenas de Paraguay, como una de las estrategias de conversión utilizadas por los misioneros católicos. Canessa (2012: 185) reporta que el catastro del año 1922 del pueblo indígena donde efectuó su investigación de campo, en los Andes bolivianos, menciona una "cancha de football" (nótese el uso de la palabra en inglés y no "fútbol").

En la región de Otavalo, un campeonato de fútbol que reúne equipos mayoritariamente formados por indígenas se organiza desde el año 1961 en la comunidad de Agato durante el periodo de carnaval. Vale decir que este deporte se jugaba antes de dicha fecha en esta área. Sin embargo, aparte del antropólogo indígena otavalo Males (1985) ${ }^{8}$ y de unas alusiones de Colloredo-Mansfeld (1999), ;no he podido encontrar más información sobre el fútbol en Otavalo en los estudios publicados en el siglo XX dentro de lo que pude consultar! Parsons (1945: 53) habla brevemente de tres juegos de pelota en la comunidad de Peguche, cercana a la ciudad de Otavalo, que ella observó en su trabajo de campo en 1940 y $1941^{9}$. Sin embargo, ninguno hace referencia al fútbol; pues se juegan con las manos. Kowii (2006: 64-65), intelectual y escritor otavalo, señala también dos juegos de pelota con manos sin contextualizarlos temporalmente.

Con el fin de aclarar un poco la llegada del fútbol en el medio indígena de Otavalo, busqué, en enero del 2014, a personas que habían participado en el primer campeonato de Agato en $1961^{10}$. Gracias a la ayuda de un conocido, tuve la oportunidad

Ver: http://es.wikipedia.org/wiki/Copa_América

8 Males (1985) habla del fútbol en el medio indígena, pero no se trata de una investigación sobre este deporte. Sin embargo, los datos que expone son preciosos para el tema del balompié en el contexto de Otavalo.

Lévi-Strauss evoca también juegos de pelota en Tristes Tropiques (Walker, 2013: 396).

10 También traté de tener acceso al periódico regional La Verdad en los archivos del Municipio de Ibarra —capital de la provincia de Imbabura donde Otavalo se encuentra-, pero los números más antiguos que se conservan se remontan solo a los años 1980 . 
de platicar con Cristian ${ }^{11}$ que me presentó a uno de sus compañeros de equipo de aquella época, Luís, un "mestizo"12 con quien conversé unos días después.

Cristian, de 78 años en el momento de la entrevista - nació, entonces, en 1935 o 1936 - me contó que, cuando era niño, jugaba balompié en la calle con sus amigos con una pelota de trapo, primero descalzos, más tarde con alpargatas y después con zapatillas de fútbol. Añadió que practicó este deporte, que adora, hasta los 65 años. Según Cristian y Luís, fue el maestro de la escuela, un "mestizo", quien les enseñó a jugar con una pelota de cuero que poseía. Por lo tanto, podemos remontar estos hechos a la segunda mitad de los años 40. Esta estimación temporal coincide con el desarrollo de la práctica de los indígenas que residían en Ibarra, la capital provincial de Imbabura, conocidos como "Imbayas de Ibarra", que provienen de la comunidad de Quinchuquí, vecina de Peguche y Agato, y que migraron al inicio del siglo XX a esta ciudad (Males, 1985; Kowii, 2006: 66). Los Imbayas veían los partidos de los blanco-mestizos en las canchas de la urbe y, al mirar continuamente este juego, unos jóvenes decidieron formar un equipo solo de indígenas. Crearon así el Club Deportivo Imbayas - CD Imbayas - en 1948, equipo que jugaba el campeonato regional (Males, 1985: 108). Unos interlocutores de Males (1985: 108-109) afirmaron que eran apasionados de este deporte, que se entrenaban de manera asidua, pero también que eran víctimas de racismo por parte de los blanco-mestizos en las canchas y en sus alrededores. El CD Imbayas tuvo un cierto éxito durante los años 50 - luego declinó con las nuevas generaciones-, lo que le permitió jugar partidos en todo el país y en varias ciudades colombianas (Males, 1985: 109-111; Kowii, 2006: 66).

El fútbol debía ser ya popular en el medio blanco-mestizo en esta época; la selección ecuatoriana participó por primera vez en torneos internacionales en 1938, en los juegos Bolivarianos en Bogotá y, en 1939, en el Campeonato Sudamericano en Lima (Ramírez, 2006: 41; Carrión, 2006: 72). Sin embargo, no fue vía los medios de comunicación que este deporte llegó a las comunidades indígenas, pues, como me dijo Cristian, no tenían televisión ni radio en aquel tiempo. Como ocurrió en Agato con el maestro de la escuela, fueron los blanco-mestizos quienes difundieron, la mayoría del tiempo, el fútbol en el medio indígena. Acuña Delgado $(2010: 115,117)$ afirma que, en el caso de los yanomamis de Venezuela, los misioneros, los "criollos"13 y los medios de comunicación introdujeron este deporte, y que los niños aprendieron a jugarlo primero en la escuela.

Cristian, al igual que Luís unos días después, me contó que al inicio jugaban con amigos y que luego, poco a poco, empezaron a invitar a jóvenes de comunidades vecinas para jugar partidos, como lo hacían éstos últimos con los de Agato. Más tarde se pusieron a organizar torneos, el primero en Agato en 1961, durante el carnaval. Posteriormente, se desarrolló este campeonato y poco a poco otras comunidades empezaron a organizar torneos en diferentes épocas del año. En 2011, el "Carnaval de Agato" celebró su quincuagésimo aniversario.

En cuanto a Luís, me informó que enseñó el fútbol a sus compañeros de Agato, siendo el capitán, después el entrenador, de uno de los equipos de la comunidad

11 Todos los nombres de mis interlocutores son ficticios.

12 Los otavalos suelen utilizar la categoría "mishu/a" en kichwa y "mestizo/a" en castellano para identificar a una persona que no consideran como indígena, afro-descendiente o norteamericana/europea. Coincide con la categoría "blanco-mestizo" de la literatura de ciencias sociales sobre Ecuador (Whitten 1981, 2003; ColloredoMansfeld 1999; Rahier 2014).

13 Categoría equivalente a la de "blanco-mestizo" en el caso de Venezuela. 
durante torneos. Añadió que era el único mestizo del conjunto y que en los otros equipos a veces también había mestizos. Luís me dijo que las relaciones entre los indígenas y los mestizos eran buenas. Hay que precisar, sin embargo, que él era el dueño de un ishtangu (cantina) en Agato, como me contó un amigo. Este tipo de establecimientos estaba íntimamente vinculado con la dominación blanco-mestiza ${ }^{14}$. Aparentemente por su experiencia futbolística y su estatus social, Luís era un personaje influyente a nivel local.

Como lo indica Kowii (2006: 68), las actividades deportivas se desarrollaron particularmente en la región de Otavalo a partir de la segunda mitad de los años 70 y 80 del siglo anterior. Por ejemplo, la Unión de Organizaciones Campesinas de Cotacachi (UNORCAC) empezó a organizar un campeonato intercomunitario de fútbol en 1978 en la región de Cotacachi — a unos $10 \mathrm{~km}$ de la ciudad de Otavalo-, que todavía existe hoy en día, y se estableció un campeonato de básquet para jóvenes, hombres y mujeres, desde 1983 (Kowii, 2006: 67-68). En esta época, se formaron clubes informales de deporte, particularmente de fútbol, que se dedicaban principalmente a participar en eventos puntuales.

Según varias conversaciones informales con diferentes interlocutores, el torneo del Carnaval de Agato fue el más importante hasta los años 90 en la región de Otavalo, y promovió decididamente el balompié en el medio indígena. Un equipo de la comunidad vecina de Peguche solía jugar en este campeonato, pero la rivalidad continua entre los dos poblados y ciertas tensiones que se desarrollaron durante la competencia alentaron a unos jóvenes pegucheños a organizar otro torneo: el Carnaval Peguche Tío. Este fue realizado por primera vez en 1995 y cambió de nombre en 1999 a Pawkar Raymi Peguche Tío ${ }^{15}$ (ver Kowii, 2006; Wibbelsman, 2004; Muenala, 2009). Este evento tuvo y tiene un papel importante en el desarrollo del fútbol en la región de Otavalo. Logró atraer, año tras año, a los mejores jugadores y equipos de la región, así como a refuerzos profesionales no-indígenas, gracias a la gestión, los premios, la calidad de la cancha y el aforo de su estadio. Como me indicaron varios interlocutores - ver también la entrevista del encargado de los deportes del Pawkar Raymi de Peguche 2010 en Muenala (2010) - , las fiestas de Pawkar Raymi/Carnaval - que se organizan desde entonces en varias comunidades - , particularmente la de Peguche y Agato, constituyen los principales espacios-tiempos de competencias de fútbol durante el año para los indígenas de la región.

\section{Producción de la masculinidad y "moral en acto" en la práctica del fútbol en Otavalo}

A pesar de la dificultad de encontrar fuentes históricas, podemos constatar el desarrollo de la práctica del fútbol en el medio indígena en la región de Otavalo, práctica que se arraigó con el tiempo en la zona. Hoy en día, este deporte tiene una importancia considerable entre los jóvenes, adultos y mayores. Así, la llegada y el desarrollo del fútbol en la región resultaron de conexiones más allá del medio indígena, es

\footnotetext{
14 En efecto, desde el inicio de la colonia hasta años recientes, los blanco-mestizos controlaban el negocio del alcohol, producto bastante consumido por los indígenas en todos los Andes, principalmente en el contexto festivo (ver Saignes, 1993).

15 "Peguche tío" o "Peguche tiyu" es una expresión utilizada en kichwa para significar "pegucheño".
} 
decir, con los blanco-mestizos y con redes trasnacionales, también a través de los medios de comunicación. Principalmente en los inicios, fueron los blanco-mestizos de estatus particular - maestros, dueños de cantina - quienes tuvieron un papel importante; la desigualdad de base racial, es decir, la dominación de los blancomestizos sobre los indígenas, era particularmente fuerte durante el siglo XX y jugó seguramente un rol en el proceso. Este aspecto sobrepasa el alcance de este artículo y deja un campo de estudio para trabajos posteriores.

El fútbol en Otavalo ha generado formas de sociabilidad a través del aprendizaje de habilidades, es decir, reglas y técnicas del cuerpo que no tienen un anclaje "cultural", sino que tienen una dimensión trasnacional, como lo veremos todavía más adelante. Los indígenas se apropiaron de este deporte, generando pasión en algunos de ellos. Por consiguiente, el fútbol se ha convertido en una práctica familiar, sin que el origen "cultural" o "geográfico" sea pertinente en su aprehensión.

En esta sección, me interesaré por las dimensiones de género y moral que la apropiación de esta práctica deportiva genera en Otavalo. Tiene sus particularidades, ligadas a la producción de la "localidad" — "locality" — del medio indígena otavaleño, pero estos aspectos se conectan con dimensiones morales y de género trasnacionales con relación al fútbol, lo que resalta una vez más la conectividad de los otavalos más allá de su región y del territorio nacional.

A pesar de que este deporte, a nivel mundial, está siendo apropiado cada vez más por las mujeres, se ha constituido históricamente como una práctica masculina y productora de masculinidad (ver Miller, 2007; Wittersheim, 2014: 21; para el deporte en general, ver Connell, 2005). Este aspecto es también pertinente en el caso indígena de Otavalo. Aunque de vez en cuando las chicas jueguen, se trata esencialmente de una práctica masculina. Observé unos partidos y torneos organizados para las muchachas, efectuados en canchas pequeñas con una pelota de tamaño reducido, variación jugada igualmente por muchachos y que es llamada "indoor" en Ecuador - aunque no se practica en un lugar cerrado- . Sin embargo, la UNORCAC ha comenzado a organizar un campeonato femenino en canchas convencionales desde el año 2000 en la región de Cotacachi (Kowii, 2006: 67). En el año 2010, por primera vez un torneo de fútbol femenino tuvo lugar en el Pawkar Raymi de Peguche, en el mismo estadio que los hombres (Muenala, 2010), sin darle continuidad los años posteriores. Las chicas se dedican más al básquet; de hecho, el Pawkar Raymi de Peguche organiza desde sus inicios un torneo de este deporte para ellas (Muenala, 2009) ${ }^{16}$.

Los jóvenes se consagran al deporte principalmente los domingos. Según mis observaciones (y mi participación), los espacios futboleros y de ecuavoly son monopolizados por los varones, mientras las canchas de básquet son mixtas constituyéndose en sitios de encuentro entre chicas y chicos, quienes visten su mejor atuendo - las primeras con la ropa indígena, los segundos con gorras, camisetas, jeans y zapatos a la moda- .

Por lo tanto, el fútbol constituye un signo y una experiencia colectiva de masculinidad; contribuye a producirla por técnicas del cuerpo - performance y trabajo sobre éste, con el propósito de diferenciarlo del femenino-. Este aspecto puede ser generalizado a un nivel global, sin excluir la posibilidad para las mujeres de desafiarlo, adquiriendo habilidades en su práctica (ver el concepto de "masculinidad hege-

16 En esta fiesta, la competición de ciclismo tiene también una categoría para mujeres, mientras no es el caso del fútbol y del ecuavoly - tipo de voleibol ecuatoriano-. 
mónica" de Connell, 2005 [1995]: 76). Este deporte, así como el ecuavoly, se efectúa en espacios-tiempos donde las mujeres están generalmente ausentes, contribuyendo de manera significativa al desarrollo de una sociabilidad masculina. Sin embargo, en otras partes de los Andes, los partidos de fútbol entre mujeres indígenas "en pollera" son más frecuentes (Canessa, comunicación personal). Según Acuña Delgado (2010: 124), para los yanomamis de Venezuela, y según Bonifacio (2011), para los maskoys del Alto-Paraguay, el fútbol es también una práctica principalmente masculina, mientras que el voleibol es mixto.

En cuanto al aspecto moral, en el medio indígena de Otavalo, la práctica del deporte en general - y del fútbol en particular - se opone al alcohol. Como lo destaqué en mi investigación sobre fiestas (Voirol, 2016), el consumo excesivo de alcohol constituye un ingrediente fundamental en las fiestas indígenas y en otras situaciones de sociabilidad, elemento que involucra principalmente a los hombres católicos. En Otavalo se juega fútbol sin consumir alcohol; se juega o se toma. Uno de los propósitos de la organización del campeonato de la UNORCAC en 1978 era reducir el consumo de alcohol entre los jóvenes (Kowii, 2006: 67). El reglamento del Pawkar Raymi de Peguche prohíbe explícitamente su consumo por los jugadores y por el staff de los equipos, antes y durante un partido. Así, en los espacios de juego (los domingos, por ejemplo), no se ve a los participantes beber alcohol.

Esta dimensión moral es particularmente explícita en los discursos de los evangélicos y los mormones ${ }^{17}$. En efecto, el alcohol constituye un emblema alrededor del cual han hecho surgir un problema moral que les permite identificarse colectivamente (Latour, 2005); no lo consumen por ser moralmente dañino - en las fiestas lo reemplazan por gaseosas-, oponiéndose de esta manera a los católicos. En discusiones informales y durante entrevistas con miembros de estas iglesias se destacó que el fútbol y el deporte en general son prácticas alentadas por ellos. Como me dijeron Cristian, quien es evangélico, y Pepe, un mormón de Peguche, el deporte es sano y bueno para la salud: "El deporte es sano para la humanidad" (Pepe). Ricardo, el hijo de Pepe, me habló de una "ley de sabiduría" aprendida en el marco de la iglesia mormona que evoca particularmente la preocupación por la salud: "cuidar nuestro cuerpo, no tomar, no fumar".

Durante una entrevista en 2010 acerca del Killa Raymi - "Fiesta de la Luna"que se organiza en el barrio Atahualpa de Peguche desde el año 2009, Pepe, uno de sus fundadores, me explicó las razones por las que para él es positivo que una actividad deportiva, un torneo de ecuavoly en este caso, esté en el centro de la fiesta. Me dijo que, en el barrio, no había lugares para practicar deporte, de manera que la gente no se dedicara "al vicio". Añadió: "En vez de estar tomando chicha o emborrachando [sic], yo creo que el deporte ha sido bueno, y por eso ya, ya hemos creado... como en el Pawkar que dice [sic], con el deporte ya vienen otras áreas". Hace alusión, en el caso del Killa Raymi, a la noche de conciertos realizada al final del evento ese año - con grupos locales de música andina y con una banda afro-ecuatoriana- y a proyectos de revitalización, en el marco de esta fiesta, de aspectos de "su propia cultura" como la gastronomía. Como en el caso del Pawkar Raymi, el deporte llevó a organizar también eventos culturales, que son considerados como positivos por Pepe.

17 En el distrito donde se encuentran Peguche y Agato, los miembros de estas dos iglesias constituyen aproximadamente $25 \%$ de la población, el resto es de confesión católica. 
Para promover el deporte y el ideal de cuerpo sano, la iglesia evangélica de Peguche suele organizar en Navidad actividades deportivas - carrera y ecuavoly - para los niños y adolescentes, como una manera de inculcarles esta moral desde temprana edad.

Por lo tanto, el deporte aparece explícitamente en el caso de mis interlocutores evangélicos y mormones como una práctica sana para el cuerpo y una alternativa al consumo de alcohol y "al vicio", pues el exceso de esta sustancia, según ellos, daña la salud y lleva a la violencia o a la sexualidad desenfrenada. El deporte y el fútbol permiten controlar los cuerpos y evitar que "se desvíen" de lo que es considerado moralmente "bueno". Estos discursos constituyen una moral reflexiva, una forma de "public discourse of morality" según los términos de Zigon (2009: 259-260).

En la práctica, el comportamiento hacia el alcohol difiere, en efecto, entre los evangélicos y mormones, por un lado, y los católicos, por otro. Los primeros tienden a no consumirlo, mientras para los segundos su ingestión es adecuada y esperada por sus pares, hasta incitada por ellos, en las fiestas - pero no en el almuerzo, por ejemplo. Sin embargo, esta diferencia desaparece en el caso del deporte; su práctica misma excluye el consumo de alcohol, incluso en el caso de los católicos. Este aspecto constituye una dimensión moral de la práctica del deporte, que se diferencia de la de los blanco-mestizos, por ejemplo, donde se puede observar la presencia de alcohol en los espacios de juego. Inspirándome en la "ordinary ethics" de Lambek (2010) y Das (2012), así como en la "enacted morality" de Zigon (2009), esta actitud hacia el alcohol forma parte de lo que llamo "moral en acto", es decir una moral que surge de la práctica misma, poniendo en juego, en el caso del fútbol en Otavalo, el cuidado y un tratamiento específico del cuerpo, excluyendo el alcohol, que es considerado como algo que impide la buena práctica de este deporte. Hay que entender este tipo de "moral" como un ideal, como una guía para la acción, para un comportamiento adecuado y esperado y no como algo determinista, que la gente hace necesariamente. Por lo tanto, la pluralidad de la "moral en acto" observable, por ejemplo, en el marco festivo, entre los evangélicos/mormones y los católicos, no es pertinente en la práctica del deporte. De esta manera, al contrario de la actitud hacia el alcohol que hace surgir diferentes confesiones, el fútbol participa en la producción del género a través de cuerpos diferenciados — hombre/mujer - y de una moral unificada — el consumo de bebidas alcohólicas es inadecuado.

La dimensión moral del deporte y del fútbol con relación al tratamiento de los cuerpos y al control del ocio no está ausente en otros casos. En efecto, nos hace pensar en los hombres eclesiásticos británicos del fin del siglo XIX; alentaron a los jóvenes de las clases obreras a practicar deporte para alejarlos de otras actividades consideradas como "inmorales" (Miller, 2007: 4; Wittersheim, 2014: 21). Bonifacio (2011), basándose en la obra de 1954 de Susnik citada arriba, afirma que los misioneros católicos, que introdujeron el fútbol en el contexto nativo paraguayo, utilizaron este deporte para acercar a los indígenas a la vida cristiana y como alternativa a las danzas locales practicadas intensivamente durante el tiempo de ocio, que incluían el consumo excesivo de alcohol e inducían, según los religiosos, relaciones sexuales ilícitas. Dado que las reglas del fútbol son claras y establecidas, podemos pensar que la promoción de este deporte permite un cierto control del ocio; es lo que afirma por lo menos Walker (2013: 383) en el caso de la reapropiación del fútbol por el Estado. 


\section{El fútbol como reafirmación indígena: surgimiento de lo racial}

Como lo mostré en la sección precedente, la práctica del fútbol hace surgir categorías como el género. Mi tentativa de construir un histórico del balompié en una zona específica dejó vislumbrar la (re)producción de otras categorías identitarias, como las de origen "racial" de "indígenas" y "blanco-mestizos" en el caso de la aparición de este deporte en la región de Otavalo o en el de los Imbayas. De manera general, el fútbol tiene la facultad de generar reagrupamientos alrededor de diferentes tipos de categorías identitarias. Se puede observar identificaciones a nivel local, en torno a una ciudad, una región, pero también aquellas que hacen surgir una clase social ${ }^{18}$ o una pertenencia nacional (ver Da Matta, 1982; Archetti, 1995; Dávila y Londoño, 2003; Ramírez, 2006; Giulianotti, 2007; Miller, 2007; Wood, 2007; Acuña Delgado, 2010; Walker, 2013; Wittersheim 2014).

En el caso del campeonato del Pawkar Raymi de Peguche, el fútbol permite materializar un sentimiento de pertenencia comunitario, pues la mayoría de los espectadores apoyan al equipo que representa el lugar donde se vive. Por los malos resultados de la selección de Peguche en 2013, Alonso, un amigo pegucheño, me confesó estar muy triste y parecía abatido. Galo, otro amigo, me afirmó que cuando Peguche gana sus partidos, el estadio se llena para ver jugar el equipo. Además, un grupo de jóvenes de la comunidad conforma una barra; intenta alentar a la selección en todo momento, con un tambor, cantando, gritando, hasta insultando al árbitro o al adversario - reproduciendo a veces estereotipos raciales hacia los refuerzos afrodescendientes- . Esta forma de apoyo surgió a través de redes trasnacionales y es característica del imaginario de los hinchas de fútbol (Wittersheim, 2014). Dichas consideraciones resaltan un "ordinario" del fútbol que es familiar para cualquier persona que se interesa por este deporte.

Sin embargo, en la región de Otavalo, y particularmente en el contexto del campeonato del Pawkar Raymi de Peguche, el fútbol permite también pensarse como "indígena" - "runa" en kichwa-y reafirmar esta identificación. En primer lugar, pude observar que el "Mundialito indígena" constituye un escenario para su performance y su reapropiación. En efecto, según el reglamento del torneo, los equipos tienen que ser conformados por jugadores "indígenas", fuera de tres refuerzos noautóctonos - refiriendo a "blancos", "mestizos" y "negros", que, como lo mostraré abajo, son considerados como mejores jugadores-. Sin embargo, ¿cómo definir a un "indígena" y cómo aplicar el reglamento debidamente? En este reglamento, el artículo 2, llamado "de las identidades", impone tres características en dos puntos para identificar a un jugador indígena. Éstas reflejan la (auto-) definición discursiva de la indigeneidad en lo cotidiano: tener por lo menos uno de los padres nativos, el pelo largo trenzado y hablar kichwa ${ }^{19}$. Sin embargo, como lo pude constatar, esta regla no es aplicada de manera rigurosa. Efectivamente, la comisión organizadora

18 En el caso del campeonato profesional ecuatoriano, ciertos equipos — como el SC Barcelona o el CD Aucassimbolizan los sectores populares y otros — como la Liga Deportiva Universitaria o el EMELEC — las elites (Carrión, 2006: 68; ver también Bustamante, 2006: 53).

19 “Art.2.- Podrán participar en el Pawkar Raymi Peguche tío, las distintas Nacionalidades [indígenas] del Ecuador que cumplan con los siguientes requisitos:

1.- Ser indígena de Padre o Madre y poseer su identidad que es la trenza.

2.- Hablar el idioma Quichua." (documento "Reglamento para el campeonato de fútbol Pawkar Raymi 'Peguche

Tío"” del año 2011 y 2012) 
del torneo tolera a dos indígenas con pelo $\operatorname{corto}^{20}$ por equipo — que tienen que ser inscritos como tales - y pide que por lo menos los padres de los jugadores hablen el kichwa, pues, en la ciudad de Otavalo principalmente, los muchachos indígenas practican escasamente su idioma materno. Este aspecto no aparece en el reglamento de 2011, pero es explícito en el artículo 11c del de 2012.

Wilo, el encargado de la organización del torneo de fútbol en el Pawkar Raymi de Peguche en los años 2011 y 2012, me dijo en una entrevista que esta concesión al artículo 2 se hace "desde siempre" y me explicó la razón ${ }^{21}$ : "realmente en cada comunidad, en cada sector pues, hay... indígenas, hijos de indígenas que, bueno, no sé por cuestiones de moda o qué será, se han cortado el pelo y de alguna forma no se quería marginarlos". Sin embargo, esta "tolerancia" ha generado "abusos de identidad", en sus términos. Cada año, unos equipos acusan a otros de hacer jugar a un "mestizo", inscribiéndolo como "indígena con pelo corto" - mientras debería entrar en la categoría de "refuerzo" - En estos casos, los encargados del torneo investigan acerca de la "indigeneidad" de los padres del jugador incriminado, es decir su pelo, su ropa - que constituye todavía un marcador identitario fuerte ${ }^{22}$ - y su aptitud en kichwa. Si lo evalúan como no-autóctono, lo descalifican y hacen perder los puntos ganados por su equipo en el partido que generó dicha acusación.

Por consiguiente, la concepción de "indígena" en el caso de este torneo no es realmente de tipo "cultural" — "étnico"-, sino de base "racial". En efecto, reproduce categorías para pensar la diferencia proviniendo del periodo colonial y que descansan ideas sobre cuerpos y filiación, así como sobre una jerarquización de los grupos raciales. Estas categorías surgieron del proyecto de dominación de los europeos durante los procesos de colonización (Wade, 2015) y siguieron siendo pertinentes en la construcción de los nacionalismos latinoamericanos, como lo veremos más abajo.

En el caso del "Mundialito", los "indígenas" se piensan como diferentes a los blanco-mestizos y a los afro-descendientes que, de hecho, son considerados como mejores jugadores. En este contexto, que procede de la cotidianidad otavaleña, la concepción de "indígena" resalta en primer lugar rasgos físicos, de apariencia, así como la filiación, mientras el dominio del kichwa - elemento cultural también considerado de una cierta manera- es más bien relegado a un segundo plano. Marcadores materiales como el cuidado del pelo y la indumentaria tienen por lo tanto dimensiones raciales, puesto que se vuelven pertinentes en la identificación corporal de la diferencia - mientras otras características no-, aspecto relevante en el pensamiento de la "raza" (Wade, 2015; ver también Colloredo-Mansfeld, 1999, para el caso de Otavalo). En lo cotidiano, estos dos elementos juegan un papel fundamental en la identificación de personas no conocidas, tanto por parte de indígenas como de

20 En la región de Otavalo, el pelo corto para los varones es asociado a la categoría blanco-mestiza, es decir a gente que no se identifica y que no es identificada como "indígena". Además de los términos de "mestizo" en español y "mishu" en kichwa, los otavalos utilizan también el de "mocho" o "muchu”, que significa literalmente "cortado", para designar a los blanco-mestizos. Varias personas del comité organizador del torneo hablaban de jugadores "sin pelo" para calificar a los indígenas de pelo corto, lo que demuestra la importancia del tamaño de la cabellera.

21 En las citaciones, utilizo los “...” para denotar una pausa o una hesitación de mi interlocutor.

22 La indumentaria de los otavalos que es llamada "tradicional" o "formal", les diferencia visualmente de los blanco-mestizos y hasta de otros grupos indígenas. Sin embargo, los jóvenes tienden a vestirla mucho menos, sobre todo los chicos quienes a veces sólo utilizan uno o dos elementos — como el sombrero, por ejemplo. Su pelo largo, amarrado o trenzado — para los que lo tienen así-, basta para identificarlos como "indígenas", en el contexto de la región de Otavalo por lo menos. 
blanco-mestizos. Más allá de este tipo de situaciones, otros factores contribuyen a apoyar esta racialización, por ejemplo, la intimidad del hogar, la comida y su preparación, las redes sociales (Colloredo-Mansfeld, 1999; Voirol, 2016).

En otros casos, sin embargo, como en las noches culturales del Pawkar Raymi por ejemplo, se trata de construir una "cultura" otavalo particular, por medio de la música o la danza, lo que se distancia de una concepción racial. Como lo menciona Colloredo-Mansfeld (1999), los pensamientos de la diferencia en términos raciales y culturales/étnicos compiten en Otavalo, coexisten y se influencian. Según las situaciones, una concepción es más pertinente, pero siempre está interrelacionada con la otra; por eso, utilizo el adjetivo "étnico-racial", de manera general o cuando es difícil identificar cuál de las dos concepciones predomina.

Por consiguiente, observamos en la cancha doce jugadores nativos con pelo largo -Wilo los califica de "indígenas indígenas"-, cuatro con cabello corto y seis noautóctonos. A pesar de las concesiones a nivel de la apariencia, la cancha de juego constituye un escenario de performance de la indigeneidad, más precisamente como los otavalos la piensan, reapropiándose, en este caso, de algunas características de connotaciones raciales. La mayoría de los "actores" exhiben su larga cabellera negra trenzada, reafirmando de tal manera esta identificación y la importancia del papel de este marcador.

\section{El fútbol como reproducción y desafío de la desigualdad racial en la sociedad ecuatoriana}

La práctica del fútbol en el campeonato del Pawkar Raymi hace (re)surgir identificaciones raciales y reproduce la configuración desigual heredada del período colonial. Sin embargo, a través de este deporte, los indígenas se animan a desafiarla. En primer lugar, los blanco-mestizos y los europeos, pero también los afro-descendientes, son considerados como superiores en el fútbol (para la Amazonía peruana, ver Walker, 2013: 389), como el reglamento del Mundialito indígena refleja. En efecto, Wilo me explicó durante nuestra entrevista que considera a los indígenas como "atrasados" comparados con estos otros grupos de la población, y que el objetivo del torneo de la fiesta siempre ha sido el desarrollo del nivel de los jóvenes nativos, que no tienen muchas oportunidades. Así, el papel de los refuerzos es enseñarles a jugar mejor y a elevar el nivel del fútbol en el campeonato. Wilo me dijo:

[Nosotros como] amantes del deporte, en especial del fútbol pues no, veíamos la necesidad de que... nuestros jóvenes no tenían espacio, o sea no tenían oportunidades para practicar el fútbol, que a nosotros nos gusta muchísimo. Y lastimosamente, en el medio donde vivimos pues, siempre nos han dado poca, poca... pocas oportunidades, porque realmente los que están a la cabeza de las ligas barriales, de los equipos profesionales, semi-profesionales, son [sic] la gente mestiza, y de alguna forma siempre nos han marginado a nosotros... los indios, no. Y nuestra aspiración realmente era que... empezar aquí, echar raíces, para que tarde o temprano, en un tiempo no muy lejano pues, ver a nuestros jóvenes, ver a nuestros indios jugar, que jueguen realmente, practiquen el fútbol en, ya no únicamente dentro de la comunidad, sino en las ligas mayores, en la profesional. Es nuestra aspiración, pero que, que... como esperanza, todavía no hemos perdido, pero real- 
mente hemos avanzado muy poco, muy poco. Prácticamente, esto requiere muchos recursos [...]. Nosotros, por cuestiones de trabajo, por cuestiones del hogar, no podemos estar ahí en su total... totalmente ahí dedicado a eso.

[...] Por lo menos, cuando vemos que uno o dos [indígenas] tratan de escalar a esos niveles, es como si tu hijo o tu hermano estuviera jugando, o sea, no, no... no es nada ajeno tuyo, es como si tú mismo estuvieras jugando, y te da... es motivo de orgullo, de alegría, sin que sea nada pariente tuyo, pero por ser hermano de sangre, un indio, realmente es un orgullo, esa es nuestra aspiración, como amantes del deporte pues.

Como se puede observar, Wilo resalta el contexto de discriminación y marginalización que recae sobre los indígenas - que es relevante desde la colonización española, como lo mencioné arriba, y que ha continuado bajo la república desde el inicio del siglo XIX - . Lo relaciona con el nivel bajo de fútbol de los autóctonos comparándolo con los otros grupos "raciales". Este tipo de discurso se basa sobre una experiencia vivida y enfatiza la pertinencia de la "raza" en el contexto ecuatoriano. Wilo utiliza el término "indio" para calificar a los indígenas, palabra que tiene una connotación racial peyorativa y que ya casi no es empleada - sobre todo por los nativos - excepto a veces como insulto por parte de los blanco-mestizos. La reapropiación de este término hace todavía más fuerte su sentimiento de identificación indígena, que es conceptualizada como una relación de sangre según su testimonio, lo que remite a una filiación biológica, característica del pensamiento racial. Sin embargo, Wilo no echa toda la culpa a la posición de los indígenas en el contexto y la historia nacionales, sino que es también autocrítico afirmando que ellos mismos no hacen lo suficiente para desarrollar el fútbol en el medio indígena, alejándose así de un determinismo estricto.

En 2011, entrevisté a Lizardo en su oficina en un Ministerio en Quito. Este intelectual de Peguche, que organizó una edición del Pawkar Raymi en los 90, destacó el desafío étnico-racial en el espacio nacional que constituye el deporte, en un contexto todavía dominado por el racismo:

el deporte [...] es el cómo el pueblo indígena responde los niveles de segregación social que tiene, no. O sea, "miren aquí yo también puedo jugar igual que ustedes los mestizos", no, aunque nunca logramos una calidad técnica de ese nivel, pero esa forma de respuesta hacia el racismo que hay... Son cosas que no son nuestras, el fútbol, el ecuavoly, el básquet, pero que se nos identifica como parte de la sociedad en general, no, [...]. Entonces, siempre ha sido un reto el tema deportivo y va seguir siendo un reto. Soñamos con un indígena profesional de fútbol, un deportista medalla, que sé yo, nacional, etcétera, pero esto es difícil en la medida que los niveles de segregación, los niveles de exclusión, de racismo que existen, hace que nuestra gente no tenga los apoyos, los respaldos necesarios para lograr sus niveles, entonces todavía son retos.

Lizardo añade que este tipo de apoyo, para el desarrollo de aptitudes deportivas, pero también musicales, no existe en las comunidades rurales indígenas; hay infraestructuras en las ciudades, pero estas son dominadas por los blanco-mestizos. 
La aprehensión del fútbol en estos términos reproduce una idea del espacio nacional promovida por la ideología del "mestizaje", progresista, modernista, basada en categorías raciales, que influenció el nacionalismo del Estado en el transcurso del siglo XX. Este pensamiento considera a las personas identificadas como "indígenas" y "afro-descendientes" como "retrógradas" y como un obstáculo al proyecto nacionalista; tienen que volverse "mestizos", grupo que simboliza y que es garante de la unidad nacional, lo que implica en realidad un "blanqueamiento", pues se trata de una valorización de la herencia europea (Whitten, 1981; Huarcaya, 2014; Rahier, 2014). Esta aprehensión del fútbol refleja también lo que Rahier (2008) llama el "orden espacio-racial", donde los espacios marginales - particularmente el campo- son poblados por estos individuos. El caso de los afro-descendientes puede ser ambiguo, puesto que son considerados como mejores jugadores a nivel nacional, pero el estereotipo de "fuerza física natural" es reproducido para explicar dicha aptitud (Rahier, 2008: 629).

Las diferentes declaraciones de mis dos interlocutores muestran bien las tensiones que existen alrededor del deporte. Si permite pensarse como grupo étnico-racial y surgir las discriminaciones y exclusiones raciales, representa también un desafío para pensarse dentro de la nación y como participante en el mundo contemporáneo; un deseo de "progreso" y de "modernidad" que hace eco con los discursos del mestizaje. La profesionalización simbolizaría una forma de plena integración de los otavalos a nivel nacional e internacional. Constituiría una forma de inclusión, ideal que el movimiento indígena ha buscado siempre (Cervone, 2012: 12).

Seguramente es posible, puesto que el fútbol refleja un ideal democrático y nacional (Da Matta, 1982): todos son tratados supuestamente de manera igual, las mismas reglas valen para todos (Da Matta, 1982; Walker, 2013), su práctica es barata y lo que se requiere son calidades físicas, de resistencia y tácticas (Miller, 2007: 7-8). En Sudamérica, el fútbol permitió perturbar jerarquías sociales, raciales y continentales: desde el inicio del siglo XX, los mejores jugadores brasileños y peruanos han sido afro-descendientes y varias selecciones nacionales sudamericanas han dominado las europeas en los torneos internacionales (Miller, 2007: 7-8).

\section{El fútbol como ingrediente de pertenencia nacional para los otavalos}

En las dos secciones precedentes, resalté la persistencia de la cuestión racial con relación al fútbol en Otavalo. En efecto, no se trata de culturalizar este deporte, sino de jugarlo como se hace en todo el mundo y de dominar las reglas y técnicas, de ponerse al nivel, desde un punto de vista otavalo, de los blanco-mestizos y afro-descendientes, un nivel que se evalúa trasnacionalmente. La problemática cultural no es pertinente en este caso, como lo puede ser en otros ámbitos de la vida de los otavalos - por ejemplo, como ya señalé, en las noches culturales del Pawkar Raymi-.

Como mencioné más arriba, el fútbol constituye un medio eficaz para el reagrupamiento en torno a categorías identitarias, como la de "indígena" en el caso otavalo. Varios autores ${ }^{23}$ han resaltado este hecho sobre todo en lo que concierne al sentimiento de pertenencia a la nación. A partir del inicio del siglo XX, el balompié es

23 Como mencioné arriba, ver Da Matta (1982); Archetti (1995); Dávila y Londoño (2003); Ramírez (2006); Giulianotti (2007); Miller (2007); Wood (2007); Acuña Delgado (2010); Walker (2013); Wittersheim (2014). 
reapropiado poco a poco por los sectores urbanos populares y surgen competencias internacionales donde se enfrentan selecciones nacionales, como la Copa América, las Olimpiadas y la Copa del Mundo (Archetti, 1995; Giulianotti, 2007: 40). Esto ha contribuido a crear identidades nacionales englobantes a pesar de que ambigüedades raciales se mantienen — para el Perú, ver Wood (2007); para Brasil, ver Da Matta (1982); para Argentina, ver Archetti (1995)_. En el caso ecuatoriano, la selección participa en el desarrollo del sentimiento nacional sobre todo a partir de los años 90, lo que supera momentáneamente el regionalismo — principalmente entre la parte andina y costeña- y la discriminación racial. Esto ocurre en cuanto conoce una reestructuración y empieza a desempeñar un papel de mayor importancia en las competencias internacionales — como en la Copa América 1993 y en los Mundiales 2002, 2006, 2014 - volviéndose un referente patriótico central y unificador (Ramírez, 2006; Carrión, 2006). La profesionalización desde 1950 (Bustamente, 2006: 37), así como la creación de un campeonato nacional en los 60s que toma más importancia que los torneos regionales (Ramírez, 2006: 39; Carrión, 2006: 68), tuvo su influencia en este proceso. Ante el fin del conflicto armado con el Perú en la segunda mitad de los 90, la crisis económica que empezó en 1999 y el descrédito de las instituciones estatales, el fútbol se vuelve casi el único emblema de sentimiento y orgullo nacionales, así como de integración de la diversidad social (Ramírez, 2006: 50, 53).

Hace falta añadir que el aumento del número de países calificados para el Mundial (treinta y dos desde 1998) permitió a más naciones ser representadas, alentando de esta manera el desarrollo de identidades nacionales en torno al fútbol (Miller, 2007: 15) y proyectando estos países sobre la escena internacional por los medios de comunicación, principalmente por Internet y la televisión (Carrión, 2006: 76). El Ecuador participó por primera vez en una Copa del Mundo en el año 2002 y se calificó de nuevo en 2006 y 2014. Esto ayudó a desarrollar alrededor de este deporte un sentimiento nacional fuerte que se difundió también en el medio indígena. En efecto, conozco a muchos otavalos que siguen los partidos de la selección ecuatoriana en la televisión o en el estadio y la apoyan con mucho fervor. Tuve la oportunidad de seguir en Facebook, durante el Mundial de Brasil 2014, el entusiasmo patriótico de mis amigos ecuatorianos tanto indígenas como blanco-mestizos. Poco antes del primer partido del Ecuador contra Suiza, un joven de Peguche residiendo en México posteó el siguiente texto en su muro, que manifiesta un sentimiento de pertenencia nacional:

\section{Llegó el día!!!}

Hoy aparecemos en esena $[\mathrm{sic}]$ mundial frente a Suiza, hoy debutaremos en otro mundial, con las mismas iluciones [sic] y los mismos sueños q en años anteriores... hoy tiene q ser un día histórico para todo un país q cree en esta selección, somos 14 millones de personas q alentaremos con el alma a 11 guerreros q representan las esperanzas y los sueños de un pueblo q cree q si se puede un pueblo q ya se siente orgullo [sic] de estar en Brasil 2014...

Vamos mi selección... Vamos mi Ecuador!!!

Q si se puede carajo...

Ecuador vs Suiza 11 am hora ecuador.

El fútbol, como deporte que ve enfrentarse naciones, puede generar una nueva manera de pensarse como formando parte de una de estas naciones. El Estado y 
la sociedad blanco-mestiza han tendido a pensar los indígenas fuera de la nación. Si los intelectuales indigenistas, desde el inicio del siglo XX, intentaron ubicarlos dentro de ella, lo efectuaron a través de la ideología del mestizaje -ideología que llevó a políticas públicas particulares del Estado ecuatoriano principalmente desde los años 70-, implicando el "blanqueamiento" y la asimilación, como mencioné arriba (Whitten, 1981; Huarcaya, 2014). En 1998, el Estado ecuatoriano adopta oficialmente el multiculturalismo en su nueva constitución (Rahier, 2014). Whitten (2003: 65-67) afirma que, en los años 90, militantes afro-descendientes e indígenas se identificaban no solo "étnicamente", sino también como ecuatorianos, rechazando de esta manera el mestizaje y promoviendo el multiculturalismo. En los estudios de Whitten (1981, 2003), de Stutzman (1981), de Crain (1990) o de Huarcaya (2014), se dibuja una tendencia que ve la nación desde arriba, y a los indígenas como resistentes a una nación homogénea, sin interesarse por la interpretación que se hace en el medio autóctono - lo que da una imagen de los indígenas como un grupo bien delimitado, con una "identidad" bien marcada —, ni por el punto de vista de los indígenas en lo cotidiano.

De igual manera Wibbelsman (2004: 81) y Ordóñez (2008: 83) ven el deporte en Otavalo como una actividad que genera una unificación "étnica", mientras que Cervone (2012: 130) percibe el fútbol en la provincia ecuatoriana de Chimborazo como una competición entre indígenas y blanco-mestizos. Ninguna de las tres autoras considera estas prácticas como un medio potencial de integración nacional, sino más bien como antitéticas a esta. Sin embargo, apoyando a la selección, celebrando sus triunfos y llorando los fracasos, los otavalos hacen como sus compatriotas, se vuelven parte del pueblo ecuatoriano y pueden ser potencialmente reconocidos como tales. Al contrario del servicio militar, que puede constituir un fenómeno que permite construir un sentimiento nacional para los indígenas, como lo afirma Canessa para Bolivia (2012: 223), esta manera de pensarse en la nación surge desde abajo, y hasta desde el margen. Se imagina así una nación donde todos son "ecuatorianos", sin prejuicios sobre su carácter asimilado o sobre su pertenencia a una "cultura específica", vale decir, una nación diferente a la del mestizaje y del multiculturalismo. Además, el fútbol como fuerza unificadora nacionalista — superando momentáneamente las divisiones y discriminaciones raciales, étnicas y de clases-, puede recrear la nación fuera de la construcción institucional de emblemas de referencias - como tradiciones, alta cultura, héroes e historia nacionales, entre otros-- particularmente en momentos de crisis de los organismos estatales como ocurrió en Ecuador en los años 1990 e inicio de los años 2000 (Ramírez, 2006: 35-36, 56-57; ver también para el caso colombiano Dávila y Londoño, 2003: 134-135).

Sin embargo, este proceso implica concebir la nación como una "comunidad", "independientemente de las desigualdades y de la explotación que pueden reinar", como un "compañerismo profundo, horizontal" (Anderson 2002 [1983]: 21). Este imaginario pudo ciertamente desarrollarse solo a partir del momento en el que la explotación económica y la discriminación étnico-racial disminuyeron - proceso materializado por la movilidad socioeconómica de una parte de los indígenas, por un acceso facilitado a la educación formal, por su integración en las instituciones políticas, con ministros desde los años 90 por ejemplo- . En efecto, se necesita un "deber de olvido", un olvido de esta historia de marginalización de los indígenas, olvido característico de la construcción nacional totalizante como lo afirma Bhabha (2007 [1994]). 
¿Es esta concepción inclusiva de la nación, expresada por los otavalos a través del fútbol, generalizada a todo el país? Rahier (2008) parece mostrar esto desde un punto de vista afroecuatoriano, pero añade que las palabras de los blanco-mestizos en la prensa y en Internet —durante las buenas prestaciones de la selección en el Mundial 2006 - presentan una nación que promueve la concepción del mestizaje. Por una parte, omiten a menudo precisar que la mayoría de los jugadores son afrodescendientes, manteniendo una imagen homogénea definida en términos de mestizaje exclusivo (es decir blanco-mestizo), y, por otra, cuando lo hacen, identifican a estos jugadores con estereotipos raciales refiriendo a la "fuerza natural". La alteridad de los afro-descendientes con relación a la ecuatorianeidad surge explícitamente cuando la selección pierde. Así, durante la mala prestación en la Copa América en 2015, observé un gran número de palabras racistas en las redes sociales de Internet, incluso por parte de indígenas.

Por lo tanto, el potencial del fútbol de cumplir una forma de inclusión y de reconocimiento en la práctica, yendo más allá de un debate entre asimilacionismo y multiculturalismo, es temporal y frágil; no borra necesariamente el pensamiento del mestizaje que implica el "orden espacio-racial" mencionado, como lo nota Rahier (2008) en su análisis.

\section{Conclusión}

En este artículo, intenté mostrar la complejidad de la relación entre un deporte, el fútbol, y un grupo indígena, los otavalos. Este juego ya tiene un arraigamiento fuerte en la región; no se trata de algo nuevo, pues es practicado desde los años 40 por los otavalos, formando parte de su vida cotidiana ya desde hace mucho tiempo. Las fiestas, principalmente el Carnaval de Agato y el Pawkar Raymi de Peguche, permitieron engendrar competencias regulares y desarrollar así este deporte dentro del medio indígena. Los contactos con el mundo blanco-mestizo impulsaron al inicio la reapropiación de este juego por los otavalos — principalmente por los varones - . En un segundo momento, los medios de comunicación - particularmente la televisión e Internet hoy en día-, así como la migración, han jugado también un papel fundamental en este proceso. Como lo afirma Giulianotti (2007: 38), "Televised images of international football events fill the imaginations and memories of diverse peoples". Dada la popularidad mundial del fútbol, estas imágenes crean un imaginario compuesto de individuos-estrellas, de clubes-estrellas y de naciones-estrellas. Estas entidades hacen soñar a personas - tanto indígenas como no-autóctonas - alrededor del globo a través de su (sobre-) mediatización y de las competiciones como el Mundial, las eliminatorias para este, la Champions League europea o la Copa América y la Copa Libertadores en el caso subcontinental.

Por lo tanto, el balompié constituye una práctica que enfatiza la circulación de prácticas, de ideas, de objetos - la réplica de la copa del mundo FIFA en el campeonato del Pawkar Raymi por ejemplo - y de personas, situando a los otavalos en un mundo contemporáneo conectado, aspecto que hoy en día nadie puede poner en duda (ver los trabajos de Colloredo-Mansfeld, 1999; Meish, 2002; D'Amico, 2014 [2011] por ejemplo). Interesarse por el fútbol permite "desexotizar" a los indígenas y sacarlos del confinamiento local. Su experiencia de vida no comprende solo rituales o maneras de pensar exóticos, como nos quiso hacer creer la antropología andina 
clásica que apartó de sus estudios el fútbol, y como pretenden hacer los partidarios del giro ontológico con cosmologías propias de un pueblo (ver Descola, 2005; De la Cadena, 2015, por ejemplo). Se trata también de conexiones regionales, nacionales y trasnacionales, así como de deseos de pertenecer al mundo globalizado, lo que vuelve imposible definir una manera de ver el mundo encerrada en sí misma.

Por estas razones, no se observan intenciones de "culturalización" de este deporte —o tal vez solo unos elementos (Acuña Delgado, 2010; Walker, 2013) _; ninguna "cosmología otavalo" impone una adaptación del balompié según sus principios, el objetivo de los jugadores indígenas no es crear un juego culturalmente propio, sino más bien el dominio de las reglas oficiales y trasnacionales. Se trata de imitar a los jugadores-estrellas, a través de los gestos - técnicas o cuando uno mete un gol, por ejemplo - y del vestuario — camisetas, zapatos, etc.—, de copiar a sus hinchas barras bravas - , entre otros. Esto genera pasiones en ciertos individuos — particularmente masculinos - , que no pueden ser definidos como pertenecientes a un "grupo étnico". De esta manera, este embodiment acerca los indígenas - los otavalos y otros - a sus compatriotas y a sus coetáneos a nivel planetario, y pueden igualmente ser reconocidos como tales, como parte de un mundo actual y contemporáneo.

Por otro lado, el fútbol es producido por la gente de manera local, lo que genera particularidades. Una vez más, no dependen de un determinismo estructural, ya sea de una "cultura" o de una "cosmología", sino de una práctica que se desarrolla según lo que está en juego en las situaciones - lo que comprende también, por ejemplo, la imitación de jugadores-estrellas y la reproducción de reglas, pero no necesariamente de manera idéntica — . La "moral en acto" hace surgir una oposición entre deporte y consumo de alcohol, que es moralmente "malo" en toda situación para los mormones y evangélicos y adecuado para los católicos en los contextos festivos. Esta contradicción no se observa, por ejemplo, en el caso del juego aficionado de los blancomestizos en el Ecuador.

La práctica del fútbol, particularmente en el "Mundialito" del Pawkar Raymi de Peguche, hace surgir y reproduce una idea de sociedad racializada reflejando la experiencia de los indígenas y tejiendo así una continuidad histórica con el periodo colonial y republicano. La aprehensión de esta práctica está anclada en una visión de la sociedad que proviene de la ideología del mestizaje, pero también del multiculturalismo. Sin embargo, el fútbol permite a los otavalos no solo pensarse como tales - a través de una reapropiación de elementos étnico-raciales de identificación, como el pelo largo trenzado, estableciendo reglas que los promueven-y desafiar la configuración racial desigual de su contexto nacional. El balompié les permite también, particularmente a través del sentimiento de pertenencia a la selección ecuatoriana, imaginarse como integrados a la idea de nación, reivindicando una "ecuatorianeidad", sin consideraciones raciales (mestizaje), ni culturales (multiculturalismo).

\section{Referencias bibliográficas}

Acuña Delgado, Angel (2010). "Estructura y función del fútbol entre los yanomami del Alto Orinoco". Revista Española de Antropología Americana, 40: 111-138.

Amselle, Jean-Loup; M'Bokolo, Elikia (Eds.) (1985). Au cœur de l'ethnie. Paris: La

Découverte. 
Anderson, Benedict (2002 [1983]). L'imaginaire national. Réflexions sur l'origine et l'essor du nationalisme. Paris: La Découverte \& Syros.

Archetti, Eduardo P. (1995). "Nationalisme, football et polo: tradition et créolisation dans la construction de l'Argentine moderne". Terrain, 25: 73-90.

Becker, Howard S. (1963). Outsiders. New York: The Free Press.

Bhabha, Homi K. (2007 [1994]). "DissémiNation: temps, récit et les marges de la nation moderne", en Les lieux de la culture. Une théorie postcoloniale. Paris: Payot \& Rivages, 223-266.

Bonifacio, Valentina (2011). "La pasión para la civilización. Fútbol y Volleybol como prácticas miméticas entre los Maskoy del Alto Paraguay". Suplemento antropológico, 46: 427-476.

Bustamante, Fernando (2006). "Esbozos para una historia social del fútbol ecuatoriano", en F. Carrión (Ed.), Quema de tiempo y área chica. Quito: FLACSO, 37-63.

Canessa, Andrew (2012). Intimate Indigeneities. Durham, London: Duke University Press.

Carrión, Fernando (2006). "De la foraneidad al fin de la ventriloquia del fútbol ecuatoriano", en F. Carrión (Ed.), Quema de tiempo y área chica. Quito: FLACSO, 65-77.

Cervone, Emma (2012). Long Live Atahualpa. Indigenous Politics, Justice, and Democracy in the Northern Andes. Durham, London: Duke University Press.

Colloredo-Mansfeld, Rudi (1999). The Native Leisure Class. Chicago: The University of Chicago Press.

Connell, R. W. (2005 [1995]). Masculinities. Berkeley, Los Angeles: University of California Press.

Crain, Mary (1990). "The Social Construction of National Identity in Highland Ecuador". Anthropological Quarterly, 63(1): 43-59.

D’Amico, Linda (2014 [2011]). Etnicidad y globalización: las otavaleñas en casa y en el mundo. Quito: FLACSO, Abya-Yala.

Da Matta, Roberto (1982). "Notes sur le futebol brésilien". Le Débat, 19: 68-76.

Das, Veena (2012). "Ordinary Ethics", en D. Fassin (Ed.), A Companion to Moral Anthropology. Chichester: Wiley-Blackwell, 133-149.

Dávila, Andrés; Londoño, Catalina (2003). "La nación bajo un uniforme. Fútbol e identidad nacional en Colombia 1985-2000”, en P. Alabarces (Ed.), Futbologías. Buenos Aires: CLACSO, 123-143.

De la Cadena, Marisol (2015). Earth Beings. Ecologies of Practice Across Andean Worlds. Durham, London: Duke University Press.

Descola, Philippe (2005). Par-delà nature et culture. Paris: Gallimard.

Ferraro, Emilia (2004). Reciprocidad, don y deuda. Formas y relaciones de intercambios en los Andes de Ecuador. Quito: FLACSO, Abya-Yala.

Giulianotti, Richard (2007). "Football, South America and Globalisation: Conceptual Paths", en R. Miller, L. Crolley (eds.), Football in the Americas. London: Institute for the Study of the Americas, 37-51.

Huarcaya, Sergio Miguel (2014). "Imagining Ecuadorians. Historicizing National Identity in Twentieth-Century Otavalo, Ecuador". Latin American Research Review, 49: 64-84.

Kowii, Ariruma (2006). "Etnias y fútbol en los kichwa de Imbabura: el caso de los kichwa otavalo", en F. Carrión (Ed.), El jugador número 12. Quito: FLACSO, 61-73.

Lambek, Michael (2010). "Toward an Ethics of the Act", en M. Lambek (Ed.), Ordinary Ethics. Anthropology, Language, and Action. New York: Fordham University Press, 39-63. 
Latour, Bruno (2007 [2005]). Changer de société, refaire de la sociologie. Paris: La Découverte.

Males, Antonio (1985). Historia oral de los Imbayas de Quinchuqui 1900-1960. Villamanta ayllucunapac punta causai. Quito: Abya-Yala.

Meisch, Lynn A. (2002). Andean Entrepreneurs. Austin: University of Texas Press.

Miller, Rory (2007). "Introduction: Studying Football in the Americas", en R. Miller, L. Crolley (eds.), Football in the Americas. London: Institute for the Study of the Americas, 1-34.

Moerman, Michael (1994). "Le fil d'Ariane et le filet d'Indra. Réflexions sur ethnographie, ethnicité, identité, culture et interaction”, en C. Labat, G. Vermès (eds.), Cultures ouvertes, sociétés interculturelles. Du contact à l'interaction. Paris: Éditions Fontenay/ St-Cloud, L'Harmattan, 129-146.

Muenala, Germán (2009). "De carnaval a Pawkar Raymi”. Revista Somos, 1: 12-30.

- 2010. Revista Pawkar Raymi. s.1.: Comité Central del Pawkar Raymi 2010.

Ordóñez Charpentier, Angélica (2008). "Migración transnacional de los kichwa otavalo y la fiesta del Pawkar Raymi”, en A. Torres, J. Carrasco (eds.), Al filo de la identidad. Quito, Ciudad de Panamá, Madrid: FLACSO, UNICEF TACRO, AECID, 69-89.

Parsons, Elsie Clews (1945). Peguche, Canton of Otavalo, Province of Imbabura, Ecuador. Chicago: University of Chicago Press.

Rahier, Jean Muteba (2008). "El Mundial de Fútbol 2006 y la Selección Ecuatoriana. Discurso de Alteridad en la Internet y en la Prensa". Discurso \& Sociedad, 2(3): 609641.

- 2014. Blackness in the Andes. Ethnographic Vignettes of Cultural Politics in the Time of Multiculturalism. New York: Palgrave MacMillan.

Ramírez, Jacques Paul (2006). "La selección de fútbol como nuevo símbolo de identidad nacional”, en F. Carrión (Ed.), El jugador número 12. Quito: FLACSO, 35-60.

Saignes, Thierry (1993). "Borracheras andinas. ¿Por que los indios ebrios hablan en español?", en T. Saignes (Ed.), Borrachera y memoria. Lima, La Paz: IFEA, Hisbol, 43-71.

Sanjek, Roger (2014). Ethnography in Today's World. Philadelphia: University of Pennsylvania Press.

Stutzman, Ronald (1981). “El Mestizaje: An All-Inclusive Ideology of Exclusion”, en N. Whitten (Ed.), Cultural Transformations and Ethnicity in Modern Ecuador. Urbana: University of Illinois Press, 45-94.

Voirol, Jérémie (2016). Faire la fête à Otavalo. Des pratiques ludiques et d'échange à la mise en scène de la culture dans les Andes équatoriennes. Tesis doctoral. Faculté des sciences sociales et politiques. Université de Lausanne.

Wade, Peter (2000 [1997]). Raza y etnicidad en Latinoamérica. Quito: Abya-Yala.

- 2015. Race. An Introduction. Cambridge: Cambridge University Press.

Walker, Harry (2013). "State of play: The political ontology of sport in Amazonian Peru". American Ethnologist, 40: 382-398.

Whitten, Norman (1981). "Introduction", en N. Whitten (Ed.), Cultural Transformations and Ethnicity in Modern Ecuador. Urbana: University of Illinois Press, 1-44.

- 2003. "Symbolic Inversion, the Topology of El Mestizaje, and the Spaces of Las Razas in Ecuador". Journal of Latin American Anthropology, 8: 52-85.

Wibbelsman, Michelle C. (2004). Rimarishpa Kausanchik. Dialogical Encounters: Festive Ritual Practices and the Making of the Otavalan Moral and Mythic Community. Tesis doctoral. Department of Anthropology. University of Illinois at Urbana-Champaign. 
Wittersheim, Éric (2014). Supporters du PSG. Une enquête dans les tribunes populaires du Parc des Princes. Lormont: Le Bord de l'Eau.

Wood, David (2007). " ¡Arriba Perú! The Role of Football in the Formation of a Peruvian National Culture", en R. Miller, L. Crolley (eds.), Football in the Americas. London: Institute for the Study of the Americas, 126-142.

Zigon, Jarrett (2009). "Within a Range of Possibilities: Morality and Ethics in Social Life". Ethnos, 74(2): 251-276. 